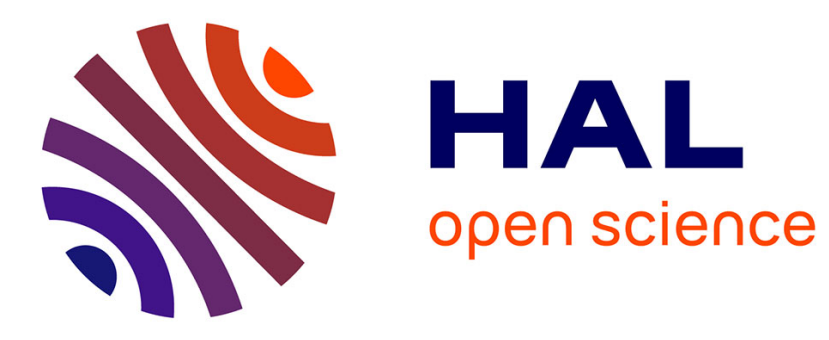

\title{
Transient Ring Opening and Closing of a Two-photon Photochromic Molecule Utilizing Energy Transfer
}

Peng Zhao, Raz Gvishi, Laura Bekere, Vladimir Khodorkovsky, David Hagan, Eric van Stryland

\section{- To cite this version:}

Peng Zhao, Raz Gvishi, Laura Bekere, Vladimir Khodorkovsky, David Hagan, et al.. Transient Ring Opening and Closing of a Two-photon Photochromic Molecule Utilizing Energy Transfer. CLEO: Science and Innovations, 2017, San Jose, France. pp.SW1M.3, 10.1364/CLEO_SI.2017.SW1M.3 . hal-03500444

\author{
HAL Id: hal-03500444 \\ https://hal.science/hal-03500444
}

Submitted on 22 Dec 2021

HAL is a multi-disciplinary open access archive for the deposit and dissemination of scientific research documents, whether they are published or not. The documents may come from teaching and research institutions in France or abroad, or from public or private research centers.
L'archive ouverte pluridisciplinaire HAL, est destinée au dépôt et à la diffusion de documents scientifiques de niveau recherche, publiés ou non, émanant des établissements d'enseignement et de recherche français ou étrangers, des laboratoires publics ou privés. 


\title{
Transient Ring Opening and Closing of a Two-photon Photochromic Molecule Utilizing Energy Transfer
}

\author{
Peng Zhao' ${ }^{1}$, Raz Gvishi ${ }^{2}$, Laura Bekere ${ }^{3}$, Vladimir Lokshin ${ }^{3}$, Vladimir Khodorkovsky ${ }^{3}$, David J. Hagan ${ }^{1}$ and \\ Eric W. Van Stryland, ${ }^{1, *}$ \\ ${ }^{I}$ CREOL, College of Optics and Photonics, University of Central Florida, Orlando, Florida 32816, USA \\ ${ }^{2}$ Applied Physics Division, Soreq NRC, Yavne 81800 Israel \\ ${ }^{3}$ Aix Marseille Université, CNRS, CINaM UMR 7325, 13288, Marseille, France \\ *ewvs@creol.ucf.edu
}

\begin{abstract}
We have developed an efficient two-photon photochromic molecule by coupling a chromene with a donating 2PA chromophore via resonance energy transfer. The essential parameters of ring opening and closing are determined from transient absorption measurements. OCIS codes: (190.4400) Nonlinear optics, materials; (190.7110) Ultrafast nonlinear optics; (190.4710) Optical nonlinearities in organic materials; (260.2160) Energy transfer;
\end{abstract}

\section{Introduction}

Photochromism of spiro-type compounds have received great interest for ultrafast photonic applications such as optical memory and switching [1]. The lower energy form usually contains a closed ring structure, which only absorbs in the ultraviolet (UV) spectral range. Upon UV irradiation, bond cleavage occurs between the $\operatorname{sp}^{3}$ carbon and oxygen atoms resulting in an open form. This is followed by cis-trans isomerization, resulting in an extended conjugation of the $\pi$-electron system exhibiting a broadband visible absorption. The open form may thermally fade back to the initial transparent closed form, with an extremely long lifetime. This back-transfer process may also be induced by absorbing a visible photon, which is known as photochemically induced ring closure [2]. The photoinduced ring opening and closing processes are usually completed within a few tens of picoseconds, accompanied by multiple transient photoproducts [2]. Ring opening can also be initiated through simultaneous absorption of two visible photons. This two-photon absorption (2PA) induced photochromism has been applied to high-density 3D optical storage [3]. Here, we are interested in the use of two-photon photochromism to enhance nonlinear absorption (NLA). Activated by 2PA, the initial transparent closed form starts to linearly absorb at the same wavelength as the 2PA excitation due to ring opening, which adds extra NLA in addition 2PA. Therefore, two-photon photochromic molecule is a potential candidate for making visible optical limiters with a truly broadband absorption.

Most photochromes are not optimized to have large 2PA cross sections (i.e. $<10 \mathrm{GM}$ ), making the 2PA induced photochromic transformation inefficient. To overcome this limitation, we have synthesized a novel bifunctional molecule (denoted PC-1 in Fig .1c) by covalently linking a photochrome, $2 \mathrm{H}$-benzo[h]chromene (2H-naphtho[1,2b]pyran) (Fig 1a), with a 2PA chromophore, 9,90-(9-(3-hydroxypropyl)-9-methyl-9H-fluorene-2,7-diyl)bis-9Hcarbazole (Fig .1b), via a 5-7 $\AA$ carboxyl bridge in an inter-orthogonal configuration [4]. Due to resonance energy transfer (RET) coupling between the 2PA donor and the photochromic acceptor, we observed $\sim 14 \times$ enhancement of the 2PA induced photochromic transformation compared to the pure photochrome. The linear absorption spectra of PC-1, 2PA donor and chromene dissolved in dichloromethane (DCM) are shown in Fig. 1d, where the relatively weaker absorption band at 370-400 $\mathrm{nm}$ and the stronger band between $300-370 \mathrm{~nm}$ originate from the photochromic moiety and 2PA donor, respectively. As a result of ring opening, a broadband absorption forms in the visible spectral range, i.e. $400-600 \mathrm{~nm}$ with a maximum at $\sim 460 \mathrm{~nm}$. In earlier studies, we have confirmed the energy transfer from fluorescence measurements of the quantum yield, excitation spectra and anisotropy [5], and the thermal dynamics were measured by studying the accumulation of open-form populations of a dye-doped sol-gel matrix [6]. Here, we perform femtosecond pump-probe measurements to resolve the transient photoproducts with the quantum yields, reaction time constants and absorption cross sections.
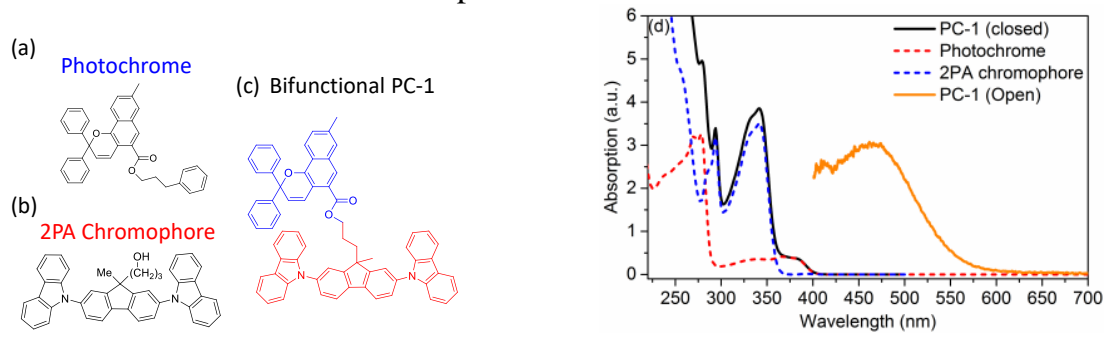

Fig .1. Molecular structures of (a) photochrome, (b) 2PA chromophore and (c) composite molecule PC-1; (d) the absorption spectra in DCM of closed- and open-form PC-1, 2PA chromophore and chromene. 


\section{Experiment and discussion}

In this experiment, the transient ring opening is studied with both one- and two-photon excitation. The wavelength of one-photon excitation (Pumpuv) is $388 \mathrm{~nm}$ which only excites the photochromic moiety of PC-1. The two-photon excitation $\left(\right.$ Pump $_{2 \mathrm{PA}}$ ) is $620 \mathrm{~nm}$, where the induced open-form absorption is negligible. To investigate photon induced ring closing, the excitation $\left(\mathrm{Pump}_{\mathrm{C}}\right)$ is chosen at $510 \mathrm{~nm}$ within the open-form absorption band. The probe wavelength is $450 \mathrm{~nm}$ for all the experiments. By applying Pump $\mathrm{C}_{\mathrm{C}}$ after Pumpuv at the time delay when the molecule is not fully opened, ring closing dynamics of the intermediate open-form transients can be measured. To avoid the accumulation of the long-lived open form, we flow the PC-1 solution through a $1 \mathrm{~mm}$ thick spectroscopic cell, so that the material is replenished within the beam size for each laser shot. Here, the repetition rate is $1 \mathrm{kHz}$.
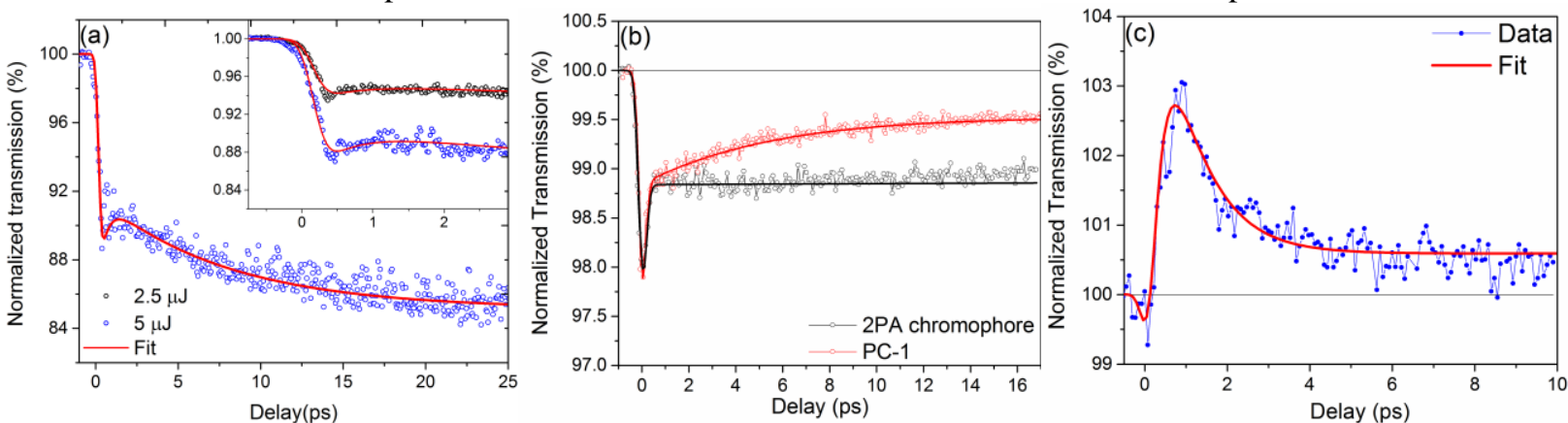

Fig .2. Data (circles) and fits (lines) of transient absorption measurements of (a) one-photon ring-opening processes (inset shows measurements with two energies within 3 ps); (b) 2PA followed by RET ring-opening processes and (c) ring-closing photochromic reactions.

The measured normalized transmission of the probe upon one-photon opening is shown in Fig. 2a, where the magnitude of the signal scales linearly with the energy of Pumpuv. After the closed form is excited, it first relaxes from a higher electronic state to a vibrational state of a lower electronic state, resulting in an excited-state absorption (ESA) at $\sim 500 \mathrm{fs}$. After overcoming the energy barrier, chemical bond cleavage between $\mathrm{sp}^{3}$ carbon and oxygen occurs for the next few picoseconds, which leads to an intermediate open form (denoted conformer A), inheriting an orthogonal parental geometry with a smaller absorption at $450 \mathrm{~nm}$ compared to the initial ESA. Finally, the slowly increasing absorption in the next $\sim 20$ ps originates from the stabilization of open form by rotation of two equal Phenyl groups farther from the naphthalene moiety. This forms the metastable open form (denoted conformer B) with extended $\pi$-electron system indicating the completion of the ring-opening process. By numerically solving the time evolution of the population in each state, we have a unique set of parameters to fit the measured transient absorption dynamics, which yields a ring-opening quantum yield of $30 \pm 7 \%$ and an absorption cross-section of 3.7 $\times 10^{-17} \mathrm{~cm}^{2}$ for conformer B. With Pump 2 PA, PC-1 and 2PA chromophore solutions are measured under identical experimental conditions (see Fig. $2 \mathrm{~b}$ ), where they show the same signal at zero delay following the cross-correlation of Pump 2 PA and probe, which originates from the nondegenerate 2PA of the 2PA chromophore (donor) only. The fits give a 2PA cross-section of $\sim 103 \mathrm{GM}$. For the 2PA chromophore, the signal remaining at longer delays is attributed to the ESA induced by degenerate $2 \mathrm{PA}$ of the Pump $2 \mathrm{PA}$ itself, which quadratically scales with the excitation energy. An ESA cross-section of $16 \times 10^{-17} \mathrm{~cm}^{2}$ is determined, which is larger than that of conformer B. However, this longlived ESA is not capable of remaining in PC-1, which instead exhibits a decay of absorption to a smaller value in $\sim 8$ ps. This maybe because coupling with the chromene via RET, the excited 2PA chromophore has another channel to relax to, initiating the ring-opening process as discussed above. Our analysis yields a RET efficiency of $76 \pm 10 \%$, in agreement with the theoretical predictions considering a Förster radius of $0.7 \mathrm{~nm}$. The ring closing dynamics are measured by applying Pump $\mathrm{C}_{\mathrm{C}}$ on conformer B solutions optically produced by illuminating the PC-1 solution reservoir with GaN LEDs emitted at $365 \mathrm{~nm}$. As shown in Fig .2c, the saturation of the open-form absorption occurs, accompanied by an intermediate closed form in $\sim 1 \mathrm{ps,} \mathrm{which} \mathrm{results} \mathrm{in} \mathrm{ring-closing} \mathrm{with} \mathrm{an} \mathrm{efficiency} \mathrm{of} 12$ $\pm 3 \%$. In another closing experiment performed on a commercially available chromene, 3,3-Diphenyl-3Hnaphtho[2,1-b]pyran, with open form produced by Pump ${ }_{U V}$, we have observed that the conformer A may have a higher closing efficiency than conformer B. We thus provide insight into the 2PA-RET photochromic reaction schemes as well as the associated essential parameters for various photonic applications, e.g. optical limiting.

\section{References}

1. H. Dürr et al, Photochromism: Molecules and Systems: Molecules and Systems (Gulf Professional Publishing, 2003).

2. J. Buback, et al, Journal of the American Chemical Society 132, 16510-16519 (2010).

3. K. D. Belfield, "FRET-based two photon three dimensional optical data storage," (Google Patents, 2011).

4. L. Bekere et al, New Journal of Chemistry 40, 1143-1148 (2016).

5. H. Hu et al, in Frontiers in Optics 2013, OSA Technical Digest (online) (Optical Society of America, 2013), FTh2C.4

6. P. Zhao et al, in CLEO: Science and Innovations, (Optical Society of America, 2014), STu3I. 6. 